\title{
ADOMIAN AND ADOMIAN-PADÉ TECHNIQUE FOR SOLVING VARIABLE COEFFICIENT VARIANT BOUSSINESQ SYSTEM
}

\author{
Ahmed J. Sabali ${ }^{\text {a, } * \text {, Saad A. Manaa }}{ }^{\text {a }}$ and Fadhil H. Easif ${ }^{\text {a }}$ \\ a Dept. of Mathematics Science, University of Zakho, Kurdistan Region - Iraq (ahmed.sabali@uoz.edu.krd)
}

Received: Jul. 2018 / Accepted: Sept., 2018 / Published: Sept., 2018

\begin{abstract}
:
In this paper, Adomian and Adomian-Padé Technique are used to find approximate solutions for the Variable-Coefficient Variant Boussinesq System, and using Adomian-Padé Technique for Debug (Remove) The Gap (Complex Root).
\end{abstract}

KEYWORDS: Adomian, Adomian-Padé, Variable-Coefficient Variant Boussinesq System, Boussinesq-Burgers (B-B).

\section{INTRODUCTION}

Most of the phenomena that arise in mathematical physics and engineering fields can be modelled by ordinary or partial differential equations. They are ubiquitous in science and engineering, as well as economics, social science, biology, business, health care, etc., which most of them are nonlinear differential equations [1]. Also, we have the mathematical representations of many physical systems $[2,3]$ It is not easy to obtain closed form solutions for such problems. In most cases, only approximate solutions either analytical ones or numerical ones can be obtained. For that reason, the nonlinear equations should be solved using special methods.

In this paper, the ADM and ADM- Padé are applied to, the Variable-Coefficient Variant Boussinesq System [4],

$$
\begin{aligned}
& u_{t}+\alpha_{1}(t) v_{x}+\beta_{1}(t) u u_{x}+\gamma_{1}(t) u_{x x}=0 \\
& v_{t}+\alpha_{2}(t) u v_{x}+\beta_{2}(t) v u_{x}+\gamma_{2}(t) v_{x x}+p(t) u_{x x x}
\end{aligned}
$$

where $\alpha_{i}(t), \beta_{i}(t), \gamma_{i}(t), i=1,2$ and $p(t)$ are arbitrary functions of $t$ and all of them are smooth functions (analytic functions) of the time $\mathrm{t}$ with $\gamma_{1}(t), \gamma_{2}(t)$ and $\mathrm{p}(\mathrm{t})$ representing different diffusion strengths, where $u=u(x, t)$ is the field of a horizontal velocity, $v=v(x, t)$ is the amplitude describing the deviation from the equilibrium position of the liquid.

Now, when we set $\alpha_{1}(t)=p(t)=-1 / 2, \beta_{1}(t)=\alpha_{2}(t)=$ $\beta_{2}(t)=2$ and $\gamma_{1}(t)=\gamma_{2}(t)=0$, then the system (1) reduces to the Boussinesq-Burgers (B-B) system $[5,6]$.The B-B equations are:

$$
\begin{aligned}
& u_{t}=-2 u u_{x}+1 / 2 v_{x}, \\
& v_{t}=1 / 2 u_{x x x}-2(u v)_{x} .
\end{aligned}
$$

The exact solitary wave solution of system (2) as in [7] are:

$$
\begin{aligned}
& u(x, t)=\frac{c k}{2}+\frac{c k}{2} \tanh \left(\frac{c k^{2} t-k x-\ln (b)}{2}\right), \\
& v(x, t)=\frac{-k^{2}}{8} \operatorname{sech}^{2}\left(\frac{k x-c k^{2} t+\ln (\mathrm{b})}{2}\right),
\end{aligned}
$$

where $c, k$ are arbitrary constants and $0<b<\infty$, with the initial conditions

$$
\begin{aligned}
& u(x, 0)=\frac{c k}{2}+\frac{c k}{2} \tanh \left(\frac{-k x-\ln (b)}{2}\right), \\
& v(x, 0)=\frac{-k^{2}}{8} \operatorname{sech}^{2}\left(\frac{k x+\ln (b)}{2}\right) .
\end{aligned}
$$

\section{BASIC IDEA OF ADOMIAN DECOMPOSITION METHOD}

We will construct the ADM and we use the method to applied on the system of equations (2).

Consider the system in an operator form

$$
\begin{gathered}
L_{t} u+L_{x} v+N_{1}(u, v)=g_{1}, \\
L_{t} v+L_{x} u+N_{2}(u, v)=g_{2},
\end{gathered}
$$

With the initial conditions:

$$
u(x, 0)=f_{1}(x), \quad v(x, 0)=f_{2}(x) .
$$

Without loss of generality, $L_{t}$ and $L_{x}$ are considered to be first order partial differential operators, $N_{1}$ and $N_{2}$ are nonlinear operators, and $g_{1}$ and $g_{2}$ are source terms.

Using the same procedure of ADM, as in [8,9]. Two recursive relations can be constructed as follows:

$$
\begin{gathered}
u_{0}(x, t)=f_{1}(x)+L_{t}^{-1} g_{1}, \\
u_{k+1}(x, t)=-L_{t}^{-1}\left(L_{x} v_{k}\right)-L_{t}^{-1}\left(A_{k}\right), \quad k \geq 0, \\
\text { and } \begin{array}{c}
v_{0}(x, t)=f_{2}(x)+L_{t}^{-1} g_{2}, \\
v_{k+1}(x, t)=-L_{t}^{-1}\left(L_{x} u_{k}\right)-L_{t}^{-1}\left(B_{k}\right), \quad k \geq 0 .
\end{array}
\end{gathered}
$$

\section{PADÉ APPROXIMANTS METHOD}

The main advantage of Padé approximation over the Taylor series approximation is that the Taylor series approximation can exhibit oscillate which may produce an approximation error bound. Moreover, Taylor series approximations can never blow-up in a finite region. To overcome these demerits, we use the Padé approximation. The Padé approximation of a function is given by a ratio of two polynomials. The coefficients of the polynomial in both the numerator and denominator using the coefficients in the Tylor series expansion of the function. The Padé approximation of a function, symbolized by $\left[\frac{P_{M}(x)}{Q_{N}(x)}\right]$ or for simplicity $\left[\frac{M}{N}\right]$, is a rational function defined by [10]

$$
\left[\frac{M}{N}\right]=\frac{p_{0}+p_{1} x+p_{2} x^{2}+\cdots+p_{M} x^{M}}{1+q_{1} x+q_{2} x^{2}+\cdots+q_{N} x^{N}},
$$

* Corresponding author

This is an open access under a CC BY-NC-SA 4.0 license (https://creativecommons.org/licenses/by-nc-sa/4.0/) 
where we considered $q_{0}=1$, and the numerator and denominator have no common factors.

There is a drawback when we truncated series solution which is correct in a very narrow region but we can't rely on this globally, for that demerit, to extend the region of solution and obtain a better accuracy and better convergence, we will use ADM together with Padé approximants (ADM-Padé technique).

The diagram below Fig. 1 shows the algorithm of ADM-Padé technique as in [11]

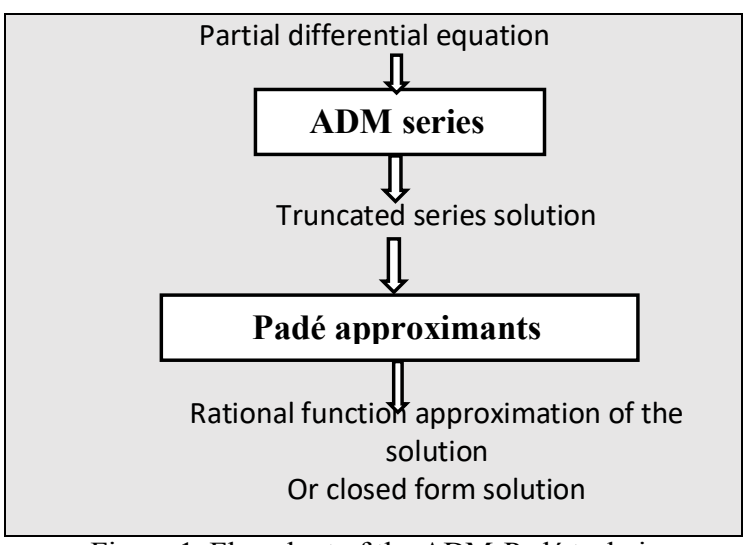

Figure 1. Flowchart of the ADM-Padé technique.

Now, when we obtain the truncated series solution of order at least $\left[\frac{M}{N}\right]$ in $t$ that we will use it to obtain Padé $\left[\frac{M}{N}\right](x, t)$ approximate solution for $u(x, t)$ as in [12] using the procedure as in $[12,13]$ we will get the ADM-Padé technique.

If we write the coefficients of $P_{M}(x)$ and $Q_{N}(x)$ as

$$
\begin{aligned}
& P_{M}(x)=p_{0}+p_{1} x+p_{2} x^{2}+\cdots+p_{M} x^{M}, \\
& Q_{N}(x)=1+q_{1} x+q_{2} x^{2}+\cdots+q_{N} x^{N} .
\end{aligned}
$$

Finally, we get such a formula of ADM-Padé technique after solving the two polynomials $P_{M}(x)$ and $Q_{N}(x)$, the results of ADM-Padé technique appear in equation (11).:

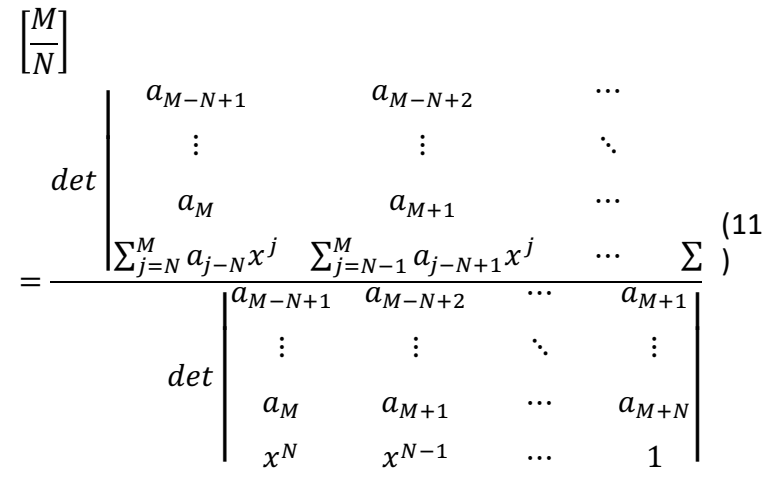

\section{APPLICATION}

In this section, we demonstrate the analysis of numerical methods [ADM \& ADM-Padé] by applying methods to the system of nonlinear partial differential Equations (2)

Note that all the numerical results for $u(x, t)$ and $v(x, t)$ that we calculate it by using ADM and ADM-Padé technique and all Figures and Tables, we used MATHEMATICA software due the simplicity and powerful manipulation.

For numerical result of system (2) and using four terms approximation solution by ADM are:

$$
u(x, t)=u_{0}(x, t)+u_{1}(x, t)+u_{2}(x, t)+u_{3}(x, t) .
$$

$$
\begin{aligned}
u(x, t)= & \frac{1}{4}\left(-\tanh \left(\frac{1}{2}(x-\log (2))\right)-1\right) \\
& -\frac{1}{16} \operatorname{sech}^{2}\left(\frac{1}{2}(x-\log (2))\right) \\
& +\frac{1}{8} \sinh ^{4}\left(\frac{1}{2}(x-\log (2))\right) \operatorname{csch}^{3}(x-\log (2)) \cdot t^{2} \\
& -\frac{1}{768} \operatorname{sech}^{4}\left(\frac{1}{2}(x-\log (2))\right)(\cosh (x-
\end{aligned}
$$

$\log (2))-2) \cdot t^{3}$

And

$$
\begin{aligned}
v(x, t)= & v_{0}(x, t)+v_{1}(x, t)+v_{2}(x, t)+v_{3}(x, t) . \\
v(x, t)= & -\frac{1}{8} \operatorname{sech}^{2}\left(\frac{1}{2}(\log (2)-x)\right) \\
& +\frac{1}{2} \sinh ^{4}\left(\frac{1}{2}(x-\log (2))\right) \operatorname{csch}^{3}(x-\log (2)) \cdot t \\
& -\frac{1}{128} \operatorname{sech}^{4}\left(\frac{1}{2}(x-\log (2))\right)(\cosh (x-\log (2)) \\
& +\frac{1}{768} \tanh ^{2}\left(\frac{1}{2}(x-\log (2))\right) \operatorname{sech}^{4}\left(\frac{1}{2}(x\right. \\
& -\log (2)))(\cosh (x-\log (2))-5) \cdot t^{3}
\end{aligned}
$$

For numerical result of system (2) and using four terms approximation, the $\left[\frac{2}{2}\right]$ order approximate solution by ADMPadé Technique are:

$$
\left.\begin{array}{l}
u(x, t)= \\
\text { and } \left.\left(\begin{array}{c}
\sinh \left(\frac{1}{2}(x-\log (2))\right)+ \\
\cosh \left(\frac{1}{2}(x-\log (2))\right)
\end{array}\right)\left(\begin{array}{c}
3\left(t^{2}+12 t+48\right) \cosh \left(\frac{1}{2}(x-\log (2))\right)+ \\
\left.t^{2}+12 t+48\right) \cosh \left(\frac{3}{2}(x-\log (2))\right)+ \\
3 t(t+4)\left(\begin{array}{c}
\sinh \left(\frac{3}{2}(x-\log (2))\right)- \\
11 \sinh \left(\frac{1}{2}(x-\log (2))\right)
\end{array}\right)
\end{array}\right)\right) \\
v(x, t)=\left(\begin{array}{c}
\left(\begin{array}{c}
-8\left(t^{2}-6\right) \cosh (x-\log (2))+ \\
\left(t^{2}+12\right) \cosh (2 x-\log (4))+ \\
9 t^{2}-24 t \sinh (x-\log (2))+ \\
6 t \sinh (2 x-\log (4))+ \\
36
\end{array}\right) \\
\frac{1}{21 t \sinh (2 x-\log (4))+30))} \\
\left(1 6 \left(143 t^{2} \cosh (x-\log (2))+\right.\right. \\
\left(24-22 t^{2}\right) \cosh (2 x-\log (4))+ \\
\left(t^{2}+12\right) \cosh (3 x-\log (8))- \\
122 t^{2}+ \\
246 t \sinh (x-\log (2))- \\
84 t \sinh (2 x-\log (4))+ \\
6 t \sinh (3 x-\log (8))+ \\
264) \cosh (x-\log (2))+ \\
\left(t^{2}-24\right) \cosh (3 x-\log (8))- \\
8\left(\left(5 t^{2}+6\right) \cosh (2 x-\log (4))+\right. \\
4 t^{2}+48 t \sinh (x-\log (2))- \\
132 \cosh (x-\log (2))+120))
\end{array}\right)
\end{array}\right)
$$

Now, Table 1 and 2 below shows the differences between exact solution and approximate solutions using ADM and ADM-Padé technique for $u(x, t)$ and $v(x, t)$ respectively, when $x=0.4$ [arbitrary chosen] and $t \in[0,1]$. 
Table 1.

\begin{tabular}{|c|c|c|c|}
\hline $\mathbf{u}(x, t)$ & Exact & ADM & $\begin{array}{l}\text { ADM- } \\
\text { Padé }\left[\frac{2}{2}\right]\end{array}$ \\
\hline & -0.21361678 & -0.21361678 & -0.2136167 \\
\hline & -0 . & & -0.225 \\
\hline & -0.2 & -0 . & -0.2383 \\
\hline & -0 & -0 . & -0.250 \\
\hline & & & \\
\hline & -0.27576479 & -0.27582530 & -0.27584602 \\
\hline
\end{tabular}

Table 2.

\begin{tabular}{|l|l|l|l|}
\hline $\mathbf{v}(\boldsymbol{x}, \boldsymbol{t})$ & Exact & ADM & ADM- \\
$\mathbf{( 0 . 4 , 0 . 0}$ & -0.12235252 & -0.12235252 & -0.12235252 \\
$\mathbf{( 0 . 4 , 0 . 2}$ & -0.12384140 & -0.12384096 & -0.12384096 \\
$\mathbf{( 0 . 4 , 0 . 4}$ & -0.12472925 & -0.12472202 & -0.12472209 \\
$\mathbf{( 0 . 4 , 0 . 6}$ & -0.12499853 & -0.12496123 & -0.12496176 \\
$\mathbf{( 0 . 4 , 0 . 8}$ & -0.12464388 & -0.12452411 & -0.12452637 \\
$\mathbf{( 0 . 4 , 1 . 0}$ & -0.12367235 & -0.12337617 & -0.12338316 \\
\hline
\end{tabular}

Again, Table 3 and 4 shows the absolute error between the exact solution and approximate solutions by ADM, ADM-Padé technique for $u(x, t)$ and $v(x, t)$ respectively, when $x=0.4$ and $t \in[0,1]$.

Table 3.

\begin{tabular}{|c|c|c|}
\hline $\mathbf{u}(x, t)$ & Exact $-\mathrm{ADM}$ & | Exact - ADM-Padé | \\
\hline$(0.4,0.0)$ & 0 & $5.55112 \times 10-17$ \\
\hline $\begin{array}{l}(0.4 \\
0.2)\end{array}$ & $1.34874 \times 10-7$ & $1.418 \times 10-7$ \\
\hline $\begin{array}{l}(0.4 \\
0.4)\end{array}$ & $2.01176 \times 10-6$ & $2.23141 \times 10-6$ \\
\hline $\begin{array}{l}(0.4 \\
0.6)\end{array}$ & $9.42085 \times 10-6$ & $1.10718 \times 10-5$ \\
\hline $\begin{array}{l}(0.4 \\
0.8)\end{array}$ & $2.73014 \times 10-5$ & $3.41788 \times 10-5$ \\
\hline $\begin{array}{l}(0.4 \\
1.0)\end{array}$ & $6.05078 \times 10-5$ & $8.12289 \times 10-5$ \\
\hline $\begin{array}{c}\text { Least } \\
\text { square } \\
\text { error }\end{array}$ & $4.49938 \times 10-9$ & $7.89391 \times 10-9$ \\
\hline
\end{tabular}

\begin{tabular}{|c|c|c|}
\multicolumn{3}{c}{ Table 4.} \\
\hline $\mathbf{v}(\boldsymbol{x}, \boldsymbol{t})$ & | Exact - ADM | & $\begin{array}{c}\text { | Exact - ADM- } \\
\text { Padé | }\end{array}$ \\
$(\mathbf{0 . 4 , 0 . 0 )}$ & 0 & 0 \\
$(\mathbf{0 . 4}, \mathbf{0 . 2})$ & $4.41678 \times 10^{-7}$ & $4.39585 \times 10^{-7}$ \\
$(\mathbf{0 . 4}, \mathbf{0 . 4})$ & $7.22664 \times 10^{-6}$ & $7.15849 \times 10^{-6}$ \\
$(\mathbf{0 . 4 , 0 . 6 )}$ & $3.72929 \times 10^{-5}$ & $3.67666 \times 10^{-5}$ \\
$(\mathbf{0 . 4 , 0 . 8 )}$ & $1.19764 \times 10^{-4}$ & $1.1751 \times 10^{-4}$ \\
$(\mathbf{0 . 4}, \mathbf{1 . 0})$ & $2.96173 \times 10^{-4}$ & $2.89186 \times 10^{-4}$ \\
$\begin{array}{c}\text { Least square } \\
\text { error }\end{array}$ & $\mathbf{1 . 0 3 5 0 5}$ & $9.88401 \times 10^{-8}$ \\
\hline
\end{tabular}

Also, the Fig. 2, Fig. 3 And Fig. 4 Below are the surfaces for the exact solution of B.B system, ADM and ADM-Padé technique respectively, when $x \in[-10,10]$ and $t \in[0,1]$.

Furthermore, Fig. 5(a) and Fig. 6(a) are the Zoom of the part that appear like the Gap [due the complex root] in the middle of the surfaces for the surface that solved numerically by ADM, we found that using ADM-Padé Technique are overcome these demerits and the Gap is disappeared as shown in Fig. 5(b) and Fig. 6(b) for $u(x, t)$ and $v(x, t)$.

With taking into consideration the value of this Gap [Complex root], we found it by using MATHEMATICA software, for $u(x, t)$ direction the value was:

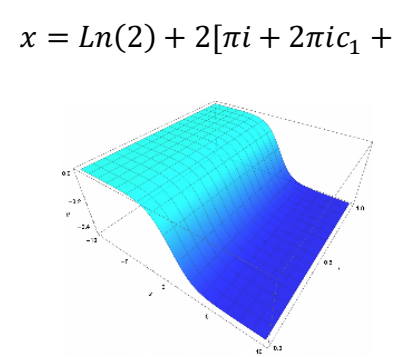

(a) $u(x, t)$

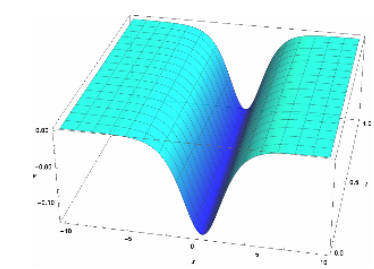

(b) $v(x, t)$
Fig. 2 The surfaces of exact solutions.

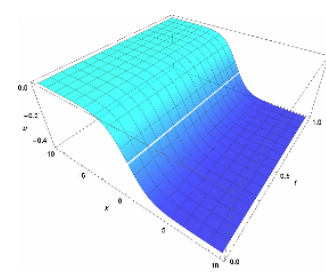

(a) $u(x, t)$

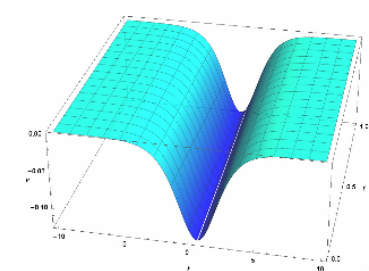

(b) $v(x, t)$
Fig. 3 The surfaces of ADM solutions.

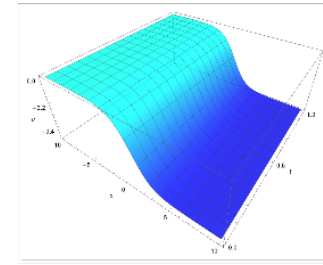

(a) $u(x, t)$

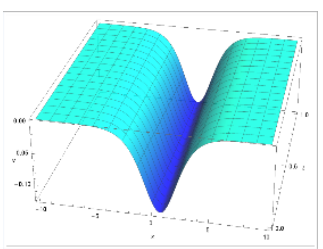

(b) $v(x, t)$
Fig. 4 The surfaces of ADM-Padé

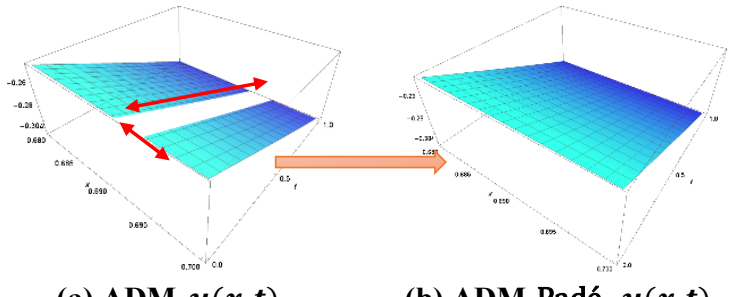

(a) ADM $u(x, t)$

(b) ADM-Padé $u(x, t)$

Fig. 5 The zoom surfaces

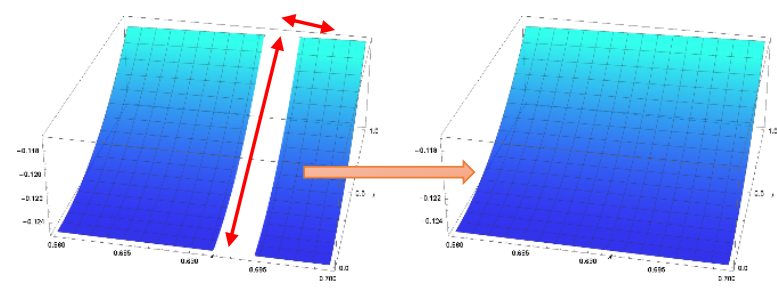

(a) ADM $v(x, t)$

(b) ADM-Padé $v(x, t)$

Fig. 6 The zoom surfaces

And for $v(x, t)$ direction the value was:

$x=\operatorname{Ln}(2)+2\left[2 \pi i c_{1}+\pi i+\frac{1}{2} \operatorname{Ln}(5-2 \sqrt{6})\right]$, where $c_{1} \in \mathbb{Z}$

The Curves in Fig. 7 and Fig. 8 shows us how the ADM and ADM-Padé curves is close to the exact solution curve, when $x=\mathbf{0 . 4}$ and $t \in[0,1]$, but as $t$ increase we see the ADM curve blow up and diverges from the exact solution curve while ADM-Padé curve preserve his path with the exact solution curve. 


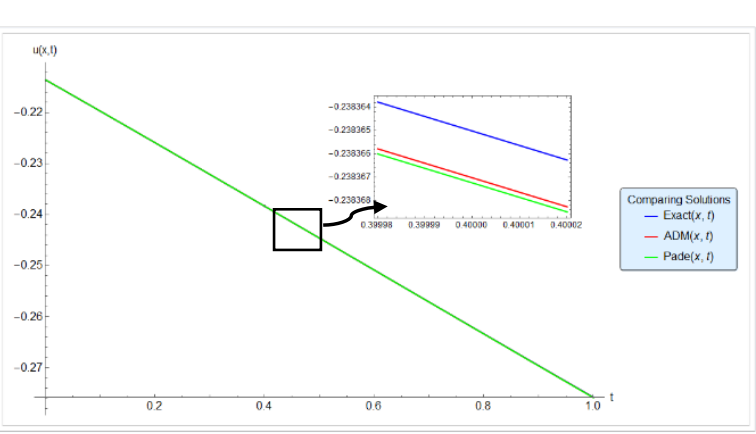

Figure 7. The curves of $u(x, t)$.

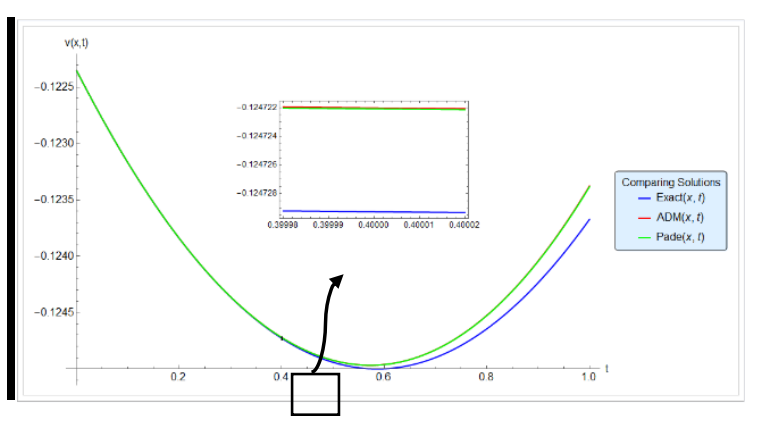

Figure 8. The curves of $v(x, t)$.

Finally, the Bar chart in Fig. 9.and Fig. 10. Show that the comparison between least square error between ADM and ADM-Padé technique of $u(x, t)$ and $v(x, t)$ respectively when $x=0.4$ and $t \in[0,1]$, of course the Less one will be better and accurate value which tends to the exact solutions curve.

\section{CONCLUSION}

In this paper the Variable-Coefficient Variant Boussinesq system was solved numerically by using Adomian Decomposition Method, Adomian-Padé technique, as an example we took the (B-B) equation and we found that $\mathrm{ADM}$ is more accurate than ADM-Padé for $u(x, t)$ direction as shown in Tables $\{1,3\}$ and Fig. 9, while ADM-Padé is more accurate than ADM for $v(x, t)$ direction as shown in Tables $\{2,4\}$ and Fig. 10. [We want to emphasize here that the values is different for $u(x, t)$ and $v(x, t)$ directions when we calculated at this specific interval of $t \in[0,1]$, but as we mentioned above when we increased $t$ the tables $\{1,2,3$ and 4$\}$, Fig. 7, Fig. 8 and Bar Charts $\{9,10\}$ will be tilts to the ADM-Padé technique due the blow up of ADM curve as $t$ increased. In general, ADM-Padé technique is more efficient and stable than ADM when $t$ increased. A point of view, this conclusion satisfied to solve this type of System of Non-Linear Partial Differential Equations]. Moreover, ADM-Padé technique Debug(Remove) The Gap (Complex Root) as shown in Fig. 4, Fig. 5(b) and Fig. 6(b) that appear after solving the equation by using $\mathrm{ADM}$ as shown in Fig. 3, Fig. 5(a) and Fig. 6(a), the work we look forward to and develop to include all types of equations that contain the complex roots after solving it by numerical methods to avoid such a kind of Gap (Complex Root) that appear in surfaces. In addition, to use the ADM-Padé technique with all types of equations and numerical methods.
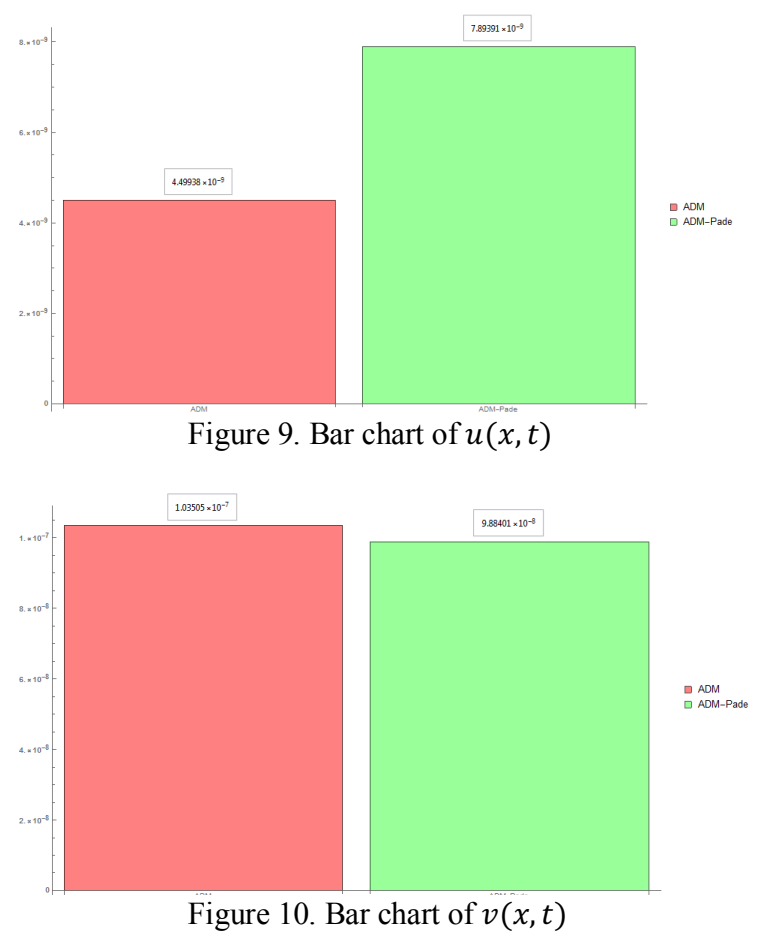

\section{REFERENCES}

[1] F. H. Easif, "Adomain Decomposition Method for Solving Non Linear Partial Differential Equations," IOSR Journal of Mathematics, vol. 10 , pp. 60-66, Sep.-Oct. (2014).

[2] Mikhailov, M. D. and Ozisik, M. N, "Unified Analysis and Solutions of Heat and Mass Diffusion, " Dover Publications, (1984).

[3] Swartz, E. T. and Pohl, R. O., "Thermal Resistance at Interfaces, " Applied Physics Letters, Vol. 51, No. 26, pp. 2200-2202, (1987).

[4] Singh, K. and Gupta, R. K., "Exact Solutions of a Variant Boussinesq System, "International Journal of Engineering Science, Vol. 44, No. 18-19, pp. 1256-1268, (2006).

[5] Mei, J. and Ma, Z., "N-Fold Darboux Transformation and MultiSoliton Solutions for the Classical Boussinesq-Burgers System, " Applied Mathematics and Computation, Vol. 219, No. 11, pp. 61636169, (2013),

[6] Wang, Y.-H., "CTE Method to the Interaction Solutions of Boussinesq-Burgers Equations, " Applied Mathematics Letters, Vol. 38 , pp. 100-105, (2014).

[7] Gupta, A. K. and Saha Ray, S, "Comparison between Homotopy Perturbation Method and Optimal Homotopy Asymptotic Method for the Soliton Solutions of Boussinesq-Burger Equations," Computers \& Fluids, Vol. 103, No., pp. 34-41, (2014).

[8] F. H. Easif, S. A. Manaa, and D. Mekaeel, "Adomain Decomposition Method for $\emptyset 4$ Klein Gordon Equation," International Journal of Engineering Inventions, vol. 2, pp. 37-42, (2013).

[9] Abderrahmane AKKOUCHE, Ahmed MAIDI, and M. AIDENE, "Solving Optimal Control Problems by Variational Approach Based on the Adomian's Decomposition Method," 3rd International Conference on Systems and Control, pp. 218-223, October, (2013).

[10]M. A. Mohamed and M. Torky, "A comparative study of numerical methods for solving the generalized Ito system," Journal of the Egyptian Mathematical Society, vol. 22, pp. 102-114, April (2014).

[11]T. A. Abassy, M. A. EI-Tawil, and H. K. Saleh, "The Solution of KdV and $\mathrm{mKdV}$ Equations Using Adomian Pade Approximation," International Journal of Nonlinear Sciences and Numerical Simulation, vol. 5(4), pp. 327-339, (2004).

[12]T. A. Abassy, M. A. El-Tawil, and H. K. Saleh, "The solution of Burgers' and good Boussinesq equations using ADM-Padé technique," Chaos, Solitons \& Fractals, vol. 32, pp. 1008-1026, 5, (2007).

[13] M. A. Mohamed and M. S. Torky, "Numerical Solution of Nonlinear System of Partial Differential Equations by the Laplace Decomposition Method and the Pade Approximation," American journal of computational mathematics, vol. 3, pp. 175-184, (2013). 